\title{
Argumentation et Analyse du discours : perspectives théoriques et découpages disciplinaires
}

Argumentation and Discourse Analysis: Theoretical perspectives and disciplinary boundaries

\section{Ruth Amossy}

\section{OpenEdition}

\section{Journals}

Édition électronique

URL : http://journals.openedition.org/aad/200

DOI : $10.4000 /$ aad. 200

ISSN : 1565-8961

\section{Éditeur}

Université de Tel-Aviv

\section{Référence électronique}

Ruth Amossy, "Argumentation et Analyse du discours : perspectives théoriques et découpages disciplinaires ", Argumentation et Analyse du Discours [En ligne], 1 | 2008, mis en ligne le 06 septembre 2008, consulté le 10 décembre 2020. URL : http://journals.openedition.org/aad/200 ; DOI : https:// doi.org/10.4000/aad.200

Ce document a été généré automatiquement le 10 décembre 2020.

\section{(c) $(1) \subseteq$}

Argumentation \& analyse du discours est mis à disposition selon les termes de la licence Creative Commons Attribution - Pas d'Utilisation Commerciale - Pas de Modification 4.0 International. 


\title{
Argumentation et Analyse du discours : perspectives théoriques et découpages disciplinaires
}

\author{
Argumentation and Discourse Analysis: Theoretical perspectives and \\ disciplinary boundaries
}

Ruth Amossy

\section{L'argumentation fait partie du fonctionnement discursif}

Dans la mesure où l'analyse du discours $(\mathrm{AD})$ entend décrire le fonctionnement du discours en situation, elle ne peut faire l'économie de sa dimension argumentative. Sans doute, toute prise de parole n'est-elle pas destinée à entraîner l'adhésion de l'auditoire à une thèse (Perelman et Olbrechts-Tyteca 1970 [1958]). De la conversation courante aux textes littéraires, nombreux sont les discours qui n'ont pas de visée argumentative, dans le sens où ils ne véhiculent aucune intention de persuader et n'entendent pas rallier l'allocutaire à une position clairement définie par des stratégies programmées. Cependant, la parole qui n'ambitionne pas de convaincre n'en cherche pas moins à exercer une influence en orientant des façons de voir et de penser. Déjà Benveniste définissait le discours comme " toute énonciation supposant un locuteur et un auditeur, et chez le premier l'intention d'influencer l'autre en quelque manière " (Benveniste 1974: 241-2). Cette définition, sans doute fragmentaire, a l'avantage de souligner que tout échange verbal repose sur un jeu d'influences mutuelles et sur la tentative, plus ou moins consciente et avouée, d'user de la parole pour agir sur l'autre. Elle met l'accent sur la force de la parole - perspective développée par les courants pragmatiques pour qui le dire est un faire, et par les théories interactionnistes selon lesquelles l'exercice de la parole implique normalement plusieurs participants lesquels participants exercent en permanence les uns sur les autres un réseau 
d'«influences mutuelles» : parler, c'est échanger, et c'est changer en échangeant (Kerbrat-Orecchioni 1990 : 54-55).

2 Dans les termes de Charaudeau, tout acte de langage émane d'un sujet qui gère sa relation à l'autre (principe d'altérité) de façon à l'influencer (principe d'influence) tout en devant gérer une relation dans laquelle le partenaire a son propre projet d'influence (principe de régulation) (Charaudeau $2005: 12$ ).

3 Est-ce à dire que tout discours est nécessairement argumentatif? Les positions sur le sujet divergent largement. La rhétorique classique définie comme art de persuader - et en ce sens, synonyme d'argumentation - considère que seuls certains genres de discours relèvent de son domaine. Aristote mentionne le juridique, le délibératif et l'épidictique, et si Perelman étend l'empire rhétorique à un ensemble beaucoup plus vaste qui comprend, comme il ressort de ses exemples, aussi bien le discours philosophique que la littérature, il n'en limite pas moins l'argumentation à la tentative de mobiliser les moyens du langage pour susciter l'adhésion des esprits à la thèse proposée à leur assentiment. Les théories contemporaines de l'argumentation vont dans le sens de cette restriction en distinguant nettement des autres les discours qui ont pour but d'agir sur l'auditoire par le moyen du raisonnement - du logos entendu comme parole et raison. En voici quelques exemples. Pour Oléron, l'argumentation est la démarche par laquelle une personne - ou un groupe - entreprend d'amener un auditoire à adopter une position par le recours à des présentations ou assertions arguments - qui visent à en démontrer la validité ou le bien-fondé (Oléron $1987: 4$ ). Selon Breton, «l'argumentation appartient à la famille des actions humaines qui ont pour objectif de convaincre. [...] [Sa spécificité est] de mettre en œuvre un raisonnement dans une situation de communication.» (Breton 1996: 3). Pour van Eemeren et le groupe d'Amsterdam fondateur de la pragma-dialectique, l'argumentation se définit comme

une activité verbale et sociale de la raison visant à accroître (ou à diminuer) aux yeux de l'auditeur ou du lecteur l'acceptabilité d'une position controversée en présentant une constellation de propositions destinées à justifier (ou réfuter) cette position devant un juge rationnel. (Van Eemeren \& al. 1984: 53 ; je traduis)

On peut cependant considérer, à l'instar de Grize, que

l'argumentation considère l'interlocuteur, non comme un objet à manipuler mais comme un alter ego auquel il s'agira de faire partager sa vision. Agir sur lui, c'est chercher à modifier les diverses représentations qu'on lui prête, en mettant en évidence certains aspects des choses, en en occultant d'autres, en en proposant de nouvelles (Grize 1990 : 41)

5 On passe alors à une conception plus large de l'argumentation entendue comme la tentative de modifier, d'infléchir, ou tout simplement de renforcer, par les moyens du langage, la vision des choses que se fait l'allocutaire. C'est la définition que j'ai avancée dans L'argumentation dans le discours (2006 [2000]) en élargissant celle de la nouvelle rhétorique de Perelman à la tentative de faire adhérer non seulement à une thèse, mais aussi à des façons de penser, de voir, de sentir. Cet élargissement permet à l'argumentation, prise comme synonyme de rhétorique ou art de persuader, de traiter du vaste éventail de discours aussi bien privés que publics qui circulent dans l'espace contemporain, et de revendiquer sa place dans les sciences du langage sans pour autant nécessiter, comme le suggère ici même Patrick Charaudeau, un recours à la psychologie et à la psychologie sociale ${ }^{1}$. 
6 Encore faut-il préciser ici la question de la divergence des points de vue, qui est au fondement de l'argumentation. Celle-ci ne surgit en effet que quand il peut y avoir une dissension, ou tout au moins une façon alternative d'envisager les choses. Comme le soulignait déjà Aristote, on n'argumente pas sur ce qui est évident - en l'occurrence, sur ce qui, dans une communauté donnée, paraît tomber sous le sens et se donner comme la seule réponse possible à une question. Cela ressort clairement de la définition que propose Michel Meyer :

Argumenter consiste à trouver les moyens pour provoquer une unicité de réponse, une adhésion à sa réponse auprès de l'interlocuteur, donc à supprimer l'alternative de leurs points de vue originels, c'est-à-dire la question qui incarne ces alternatives (Meyer 2005 :15).

7 Il s'agit cependant de savoir si les réponses alternatives que peut susciter cette question doivent être explicitées dans un contexte de confrontation. C'est ce que pense Christian Plantin, qui définit la «situation argumentative typique comme le développement et la confrontation de points de vue en contradiction en réponse à une même question " (Plantin 2005 : 53). Cette « mise en contradiction active des discours autour d'une même question» permet selon lui d'éviter la «dissolution de l'argumentation dans le langage » que risque d'entraîner la vision de Grize ou celle de Vignaux pour qui énoncer revient à argumenter (ibid.). A l'instar de ces derniers, et contrairement à Plantin, je considère que le discours en situation comporte en soi une tentative de faire voir les choses d'une certaine façon et d'agir sur l'autre. La position adverse n'a pas besoin d'être présentée en toutes lettres, dans la mesure où la parole, est toujours une réponse au mot de l'autre, une réaction au dit antérieur qu'elle confirme, modifie ou réfute :

toute énonciation, même sous forme écrite figée, est une réponse à quelque chose et est construite comme telle. Elle n'est qu'un maillon de la chaîne des actes de parole. Toute inscription prolonge celles qui l'ont précédée, engage une polémique avec elles, s'attend à des réactions actives de compréhension, anticipe sur celles-ci, etc. (Bakhtine-Volochinov 1977:105).

8 Dans cette perspective dialogique, l'argumentation est donc a priori dans le discours, au gré d'un continuum qui va de la confrontation explicite des thèses à la co-construction d'une réponse à une question donnée et à l'expression spontanée d'un point de vue personnel. C'est pourquoi il incombe à l'analyste de décrire les modalités de l'argumentation verbale au même titre que les autres processus langagiers, et en relation étroite avec ceux-ci.

9 Encore faut-il, pour éviter les confusions, distinguer entre la visée et la dimension argumentative. Même si de par sa nature dialogique, le discours comporte comme qualité intrinsèque la capacité d'agir sur autrui, de l'influencer, il faut différencier entre l'entreprise de persuasion programmée et la tendance de tout discours à orienter les façons de voir $\mathrm{du}$ /des partenaires. Dans le premier cas, le discours manifeste une visée argumentative : le discours électoral ou l'annonce publicitaire en constituent des exemples flagrants. Dans le second cas, il comporte simplement une dimension argumentative (Amossy 2006 [2000] : 32-34) : ainsi en va-t-il de l'article d'information qui se veut neutre, de la conversation familière ou d'une grande partie des récits fictionnels.

10 Lorsqu'il y a visée, le discours choisit une ou plusieurs modalités argumentatives ${ }^{2}$ - une structure d'échange particulière qui permet le bon fonctionnement de l'entreprise de persuasion. Parmi celles-ci, on peut mentionner la modalité démonstrative où une 
thèse est présentée par un locuteur, dans un discours monogéré ou dans un dialogue, à un auditoire dont il veut obtenir l'adhésion par les voies de la démonstration raisonnée, du raisonnement articulé appuyé sur des preuves. Ou la modalité négociée, dans laquelle les partenaires qui occupent des positions divergentes, voire conflictuelles, s'efforcent de trouver une solution commune au problème qui les divise et de parvenir à un consensus à travers le compromis; ou encore la modalité polémique caractérisée par une confrontation violente de thèses antagonistes, deux instances en désaccord total tentant d'emporter la conviction de l'autre, ou du tiers qui les écoute, en attaquant les thèses adverses.

11 Les choses se présentent différemment dans le cas de la dimension argumentative, où l'entreprise de persuasion est indirecte et souvent inavouée. Elle apparaît dans la mise en mots qu'effectue un discours dont l'objectif avoué est autre qu'argumentatif : un discours d'information, une description, une narration dont la vocation est de conter, le compte rendu d'un vécu dans un carnet de route ou un journal intime, un témoignage qui relate ce que le sujet $\mathrm{a} v \mathrm{vu}$, une conversation familière où les partenaires échangent des propos anodins qui ne visent pas à faire triompher une thèse, etc. C'est alors la façon dont le discours qui vise avant tout à informer, à décrire, à narrer, à témoigner, oriente le regard de l'allocutaire pour lui faire percevoir les choses d'une certaine façon, qu'il importe de dégager et d'analyser.

Dans tous les cas, l'argumentation est indissociable du fonctionnement global du discours, et doit de ce fait être étudiée dans le cadre de l'analyse du discours. Celle-ci permet en effet d'examiner l'inscription de l'argumentation dans la matérialité langagière et dans une situation de communication concrète.

\section{L'inscription de l'analyse argumentative dans l'AD}

13 Les traités de l'argumentation inspirés d'Aristote décrivent les grandes catégories de raisonnement: le syllogisme, l'enthymème, l'analogie. Certains proposent, comme l'ouvrage pionnier de Toulmin (1993 [1958]), des prototypes de schéma argumentatif ${ }^{3}$. D'autres établissent une taxinomie des arguments en essayant de regrouper des types d'arguments au sein de catégorisations qui varient largement. D'autres encore, comme la logique informelle, s'attachent à détecter les arguments fallacieux (les paralogismes). Dans toutes ces perspectives ${ }^{4}$, l'argumentation apparaît comme un enchaînement de propositions logiques qu'il faut dégager de la langue naturelle qui les véhicule et les travestit tout à la fois. Dès lors, cependant, que des schèmes sont reconstruits par une démarche qui résume les énoncés concrets en propositions pour les disposer dans une chaîne argumentative abstraite, le langagier fait figure d'obstacle. L'analyste s'emploie à l'élaguer pour retrouver le raisonnement qui le sous-tend. Il lui incombe de mettre à jour l'armature dont se soutient l'argumentation, le squelette caché sous la chair des mots.

14 C'est à cette approche que s'oppose une théorie de l'argumentation ancrée dans les sciences du langage. Comme le dit fortement Christian Plantin, «la langue naturelle n'est pas un obstacle mais la condition de l'argumentation» (1995: 259). Examiner celle-ci telle qu'elle s'inscrit concrètement dans le discours, au-delà de la schématisation qui restitue un raisonnement abstrait, permet de voir comment fonctionne effectivement l'entreprise de persuasion dans une situation de communication donnée. Au-delà d'une série de propositions logiques qui résument des 
contenus et les relations établies entre eux, on peut ainsi tenir compte de tout ce qui s'élabore dans l'entreprise de persuasion. Le discours argumentatif ne se déroule pas dans l'espace abstrait de la logique pure, mais dans une situation de communication où le locuteur présente son point de vue dans la langue naturelle avec toutes ses ressources, qui comprennent aussi bien l'usage des connecteurs ou des déictiques que la présupposition et l'implicite, les marques de stéréotypie, l'ambiguïté, la polysémie, la métaphore, la répétition, le rythme. C'est dans l'épaisseur de la langue que se forme et se transmet l'argumentation, et c'est à travers son usage qu'elle se met en place: l'argumentation, il ne faut pas l'oublier, n'est pas le déploiement d'un raisonnement qui se suffit à lui-même, mais un échange actuel ou virtuel - entre deux ou plusieurs partenaires qui entendent influer l'un sur l'autre.

15 A la condition de la langue naturelle s'ajoute donc une autre condition intrinsèque, celle de l'interaction au sein de laquelle un locuteur tient compte de l'allocutaire sur lequel il veut agir et au profit duquel il mobilise un ensemble de moyens linguistiques et de stratégies discursives plus ou moins programmées. L'argumentation se situe dans le cadre d'un dispositif d'énonciation où le locuteur doit s'adapter à son allocutaire, ou plus exactement à l'image qu'il s'en fait (dans les termes de Perelman, l'auditoire est toujours une construction de l'orateur). Elle suppose aussi que l'on tienne compte de la situation concrète d'énonciation : qui parle à qui, dans quel rapport de places, quel est le statut de chacun des participants, quelles sont les circonstances exactes de l'échange, quels sont le moment et le lieu où il prend place. Qui plus est, la parole se situe nécessairement dans le cadre d'un genre de discours qui occupe une place particulière dans un espace social donné et comporte ses objectifs, ses règles et ses contraintes propres.

16 Cette approche attentive à l'axe de la communication et de l'interaction (actuelle ou virtuelle) entre les participants de l'échange permet de conférer à l'analyse argumentative sa dimension institutionnelle et sociale. On passe alors du domaine des universaux qu'implique la rhétorique axée sur le logos comme raison atemporelle, au domaine du social dans sa relativité et ses variations historiques et culturelles. C'est dire que l'analyse argumentative adopte la vocation de l'AD qui consiste à appréhender «le discours comme intrication d'un texte et d'un lieu social» - si bien que son objet « n'est ni l'organisation textuelle ni la situation de communication, mais ce qui les noue à travers un dispositif d'énonciation spécifique. Ce dispositif relève à la fois du verbal et de l'institutionnel » (c'est la définition de Maingueneau dans le Dictionnaire d'AD, 2002).

C'est dans ce cadre communicationnel et socio-historique qu'il faut étudier de près la façon dont l'argumentation s'inscrit, non seulement dans la matérialité discursive (choix des termes, glissements sémantiques, connecteurs, valeur de l'implicite, etc.) mais aussi dans l'interdiscours. La façon dont le texte s'assimile la parole de l'autre par les nombreuses voies du discours rapporté, du discours direct ou de la citation à l'indirect libre, est primordiale. A cela s'ajoutent les modalités selon lesquelles il s'articule, sans nécessairement l'exhiber, sur les discours qui circulent avant ou autour de lui : l'hétérogénéité constitutive est l'un des fondements de la parole argumentative dans la mesure où celle-ci réagit nécessairement, que ce soit pour le reprendre, le modifier ou le réfuter, au mot de l'autre. Il importe donc de connaître l'essentiel de ce qui se dit ou s'écrit dans une société donnée sur le thème dont il est question. Que le locuteur ne s'y rapporte pas expressément ne signifie pas que son discours ne s'y alimente pas: le point de vue qu'il expose se situe toujours dans une constellation 
préexistante. Enfin, il faut examiner l'organisation textuelle qui détermine le déploiement de l'argumentation, et la façon dont le locuteur a choisi de disposer les éléments de son discours à l'intention de son auditoire.

C'est dans ce cadre également qu'il faut reprendre deux pôles de la rhétorique classique souvent délaissés par les théories de l'argumentation: l'ethos, ou construction d'une image de soi dans le discours (Amossy 1999), et le pathos, ou construction discursive de l'émotion que le locuteur entend provoquer dans son auditoire (Plantin 2000). En effet, les moyens discursifs que mobilise l'entreprise de persuasion relèvent aussi bien du logos que de l'ethos et du pathos, et c'est la façon dont elle les noue dans un échange concret effectué dans une situation de discours particulière qui confère à la parole sa force de persuasion. On entend couramment proclamer aujourd'hui que le logos, l'argumentation rationnelle qui entend persuader par les voies de l'argument et de la preuve, est en perte de vitesse et qu'elle est remplacée dans la sphère publique par l' ethos - l'élaboration par le locuteur d'une image favorable de sa personne destinée à lui conférer pouvoir et influence - et le pathos - discours s'adressant au sentiment de l'auditoire aux dépens de la réflexion. Quelles que soient les dérives possibles des démocraties contemporaines, il semble que les choses ne soient pas aussi simples. Les trois composantes classiques de la rhétorique aristotéliciennes continuent à être nécessaires, dans des dosages et des combinaisons variables, à l'entreprise de persuasion. Il revient à l'analyste de les déceler dans les textes et de dégager les modalités selon lesquelles elles s'allient pour parvenir à l'effet désiré. En résumé, l'analyse argumentative :

- 1. étudie l'argumentation en langue naturelle, dans la matérialité du discours, comme élément intégrant d'un fonctionnement discursif global

- 2. situe l'argumentation ainsi entendue dans une situation d'énonciation précise dont il importe de connaître tous les éléments (participants, lieu, moment, circonstances, etc.)

- 3. étudie la façon dont l'argumentation s'inscrit dans l'interdiscours en se situant par rapport à ce qui se dit avant et au moment de la prise de parole sur le mode de la reprise, de la modification, de la réfutation, de l'attaque,...

- 4. prend en compte la façon dont le logos, ou le déploiement des arguments en langue naturelle, s'allie concrètement à l'ethos, l'image de soi que l'orateur projette dans son discours, et au pathos, l'émotion qu'il veut susciter chez l'autre et qu'il se doit aussi de construire discursivement.

On voit en quoi cette approche essentiellement discursive se distingue de "l'argumentation dans la langue » initiée par les travaux d'Anscombre et Ducrot, à travers lesquels les études d'argumentation ont pénétré dans les sciences du langage, où l'approche pragmatique-sémantique continue souvent à faire autorité. Dans cette acception, l'argumentation constitue un fait de langue et non de discours. Qui plus est, elle ne relève pas de l'art de persuader. L'exclusion de la perspective rhétorique s'est radicalisée dans les récents travaux de Ducrot, poursuivis avec Marion Carel (2004), où il trace une nette ligne de partage entre ce qu'il dénomme "l'argumentation rhétorique » et "l'argumentation linguistique " $:$ la première seule relève du logos, alors que la seconde nie l'existence dans le discours d'" une argumentation rationnelle, qui serait capable de prouver, de justifier» (ibid.: 21) et considère qu'«il y a des enchaînements argumentatifs dans la signification même des mots et des énoncés dont le discours est fait» (ibid.: 28). On comprend aisément les implications de cette 
«méfiance radicale » envers le logos, qui s'oppose à la force conférée par la rhétorique au logos comme seule alternative à la violence - j'en ai parlé ailleurs et je n'y insiste pas.

\section{L'argumentation dans le discours : perspectives disciplinaires et interdisciplinaires}

Considérer l'étude de l'argumentation comme une branche de l'AD ouvre différentes directions de recherche ${ }^{5}$. La distinction essentielle est néanmoins celle qui sépare les perspectives disciplinaires des perspectives interdisciplinaires. D'un côté, on trouve les travaux qui se développent dans le cadre des sciences du langage et dont l'objectif premier est de dégager et de décrire des fonctionnements discursifs. De l'autre côté, se situent les travaux qui s'inscrivent dans le cadre d'autres disciplines: recherches en Communication, Etudes littéraires, Histoire, Sciences politiques, etc. Les instruments de l'analyse du discours et de l'argumentation y sont mis à profit pour répondre à des questionnements extra-linguistiques.

Dans tous ces cas de figure, le recours à l'analyse argumentative ne peut intervenir que si on reste dans le cadre des sciences des textes: il s'agit d'investigations visant à répondre aux questions qui se posent dans le champ de leur discipline propre, par l'examen systématique d'un discours ou d'un ensemble de discours. Cependant il faut, là également, établir une distinction entre les disciplines pour lesquelles le discours peut constituer un but en soi, et celles qui ne le prennent pas pour objet immédiat. Ainsi les sciences de la communication et les études littéraires s'interrogent sur la nature des discours qu'elles explorent. Elles peuvent se poser la question de savoir comment fonctionne l'implicite dans un article d'information ou dans un roman réaliste, décrire l'usage de la première personne dans le roman, ou définir la spécificité de l'éditorial. Ce n'est pas le cas de disciplines comme l'Histoire ou les Sciences politiques, pour lesquelles le genre de discours ou les modalités de la prise de parole ne constituent pas l'objet de l'investigation, et s'avèrent tout au plus instrumentales.

Le rapport des deux types d'approche à l'AD et à l'analyse argumentative, il faut le dire d'emblée, ne manque pas de faire problème - mais les difficultés ne sont pas du même ordre dans les deux cas. Dans le premier cas, la difficulté provient de la proximité des disciplines et de la mise en cause de leurs frontières respectives; dans le second cas, elle découle au contraire de la distance entre les disciplines et de la mise en cause de leur rapprochement.

En effet, dans la mesure où l'AD et, à sa suite, l'argumentation dans le discours, se penchent non seulement sur le fonctionnement du discours, mais sur la spécificité de discours appartenant à des champs différents, elles s'approprient les objets qu'investiguent les sciences de la communication et les études littéraires. C'est ce que Dominique Maingueneau a bien montré dans son Contre Saint-Proust (2006), comme dans l'article de ce numéro d'Argumentation et Analyse du Discours. Il argue du pouvoir des découpages institutionnels, du partage des tâches qu'ils autorisent et du refus de changement qu'ils entraînent, pour expliquer la barrière qui continue à se dresser entre les études littéraires et les études d'AD (dans le double sens de recherche et d'enseignement). En réalité, l'AD devrait, selon lui, fédérer les différents domaines qui s'attachent à l'exploration de discours différenciés, quelle qu'en soit la nature - ce qui exige de ne pas sanctifier le Texte littéraire dans sa différence prétendue irréductible. 
Effectivement, de nombreux travaux en $\mathrm{AD}$ concernent des textes littéraires et des corpus médiatiques. Les travaux de Patrick Charaudeau sur Les médias et l'information sont bien connus, et Maingueneau a lui-même publié un ouvrage intitulé Analyser les textes de communication (1998). L'ouvrage paru sous la direction de M. Burger et G. Martel, Argumentation et communication dans les médias (2005) comprend plusieurs contributions issues des sciences du langage (dont celles de P. Charaudeau, J.-M. Adam et R. Amossy). Dans le domaine de la littérature, le collectif édité par R. Amossy et D. Maingueneau à la suite d'un colloque de Cerisy, L'analyse du discours dans les études littéraires (2002), marque la tentative de rassembler les efforts poursuivis jusque-là dans le domaine de l'analyse du discours littéraire au sens fort du terme (à savoir, comme branche de l'AD). Il a trouvé une suite dans Sciences du texte et analyse de discours publié en 2005 par J.-M Adam et U. Heidmann, et dans un numéro spécial de la revue Littérature, "Analyse du discours et sociocritique » (no. 140, 2005).

Il n'en reste pas moins que cette tendance se heurte à bien des refus : celui des études littéraires, discipline anciennement instituée, qui n'entendent pas se laisser "résorber» par une branche des sciences du langage; celui des études en communication, cursus relativement neuf, qui éprouvent le désir grandissant de s'ériger en discipline différenciée. Faut-il en conclure que la barrière érigée entre les disciplines est purement institutionnelle et ne concerne que la distribution des territoires et des pouvoirs?

Sans méconnaître les pesanteurs institutionnelles et la logique des champs, il me semble que l'AD et l'analyse argumentative peuvent tantôt servir de cadre et tantôt fournir des instruments de travail, sans que cela nécessite pour autant un effacement des frontières disciplinaires. Celles-ci se maintiennent pour deux raisons majeures. La première est qu'une partie des types de travaux entrepris dans ces domaines se font à partir de matériaux et selon des démarches qui ne peuvent relever de l'AD et de l'argumentation dans le discours. Cela est évident dans les sciences historiques lorsqu'elles se fondent sur une étude de traces matérielles et d'objets qui ne sont pas d'ordre discursif, ou pour la sociologie quand elle se penche sur des statistiques démographiques ou entreprend des enquêtes sur le terrain. Mais cela est vrai aussi pour les études littéraires qui intègrent dans leur espace des analyses thématiques et psychanalytiques, ou des enquêtes biographiques, fondées sur des approches étrangères à l'analyse discursive.

La deuxième raison est que si l'AD et l'analyse argumentative sont mises à contribution dans les études de littérature ou de communication comme dans les sciences de l'homme, c'est pour répondre à des questions dont la nature et les enjeux ne relèvent pas des sciences du langage à proprement parler. Dans ce cadre, l'analyse argumentative comme branche de l'AD est confrontée à des questions qui ne portent pas sur des fonctionnements discursifs mais sur des sujets relevant d'autres types d'enquêtes. On peut, par exemple, se demander comment les journalistes israéliens se sont impliqués dans le compte rendu de la deuxième guerre du Liban, ou voir comment la presse suisse francophone pendant la Deuxième Guerre mondiale a rapporté les faits relatifs à la déportation des juifs et aux camps de la mort. De même, en littérature, on tente d'éclairer La comédie humaine en explorant l'usage de l'ironie chez Balzac (Bordas 2003), ou Les liaisons dangereuses de Laclos en analysant ses stratégies épistolaires (Siess 1998). 
27 Le problème ne réside donc pas uniquement dans un découpage institutionnel qui peut apparaître comme arbitraire. La différence revendiquée est ancrée dans la nature même du questionnement et dans la construction des problématiques qui caractérisent un domaine particulier, lors même que celui-ci est axé sur la mise en œuvre du langage. Qu'en est-il dans le cas des disciplines pour qui l'investigation des textes n'est pas un but en soi, et dont l'Histoire figurera ici le modèle emblématique? Sans doute les questions s'y posent-elles différemment. On sait que la tentative de faire converger l'investigation historique et l'AD (en l'occurrence, l'analyse du discours française née avec Pêcheux effectuée en son temps par Maldidier, Guilhaumou, Robin, etc.) n'a pas permis un rapprochement institutionnel des disciplines et s'est globalement heurtée, du point de vue des historiens, à une fin de non-recevoir. Pourquoi entreprendre dès lors un recoupement sous de nouveaux auspices, et en quoi l'AD contemporaine, délestée de ses ancrages marxiste et psychanalytique et nourrie d'analyse argumentative, pourrait-elle contribuer aux études historiques ? L'argument avancé en ce sens par les tenants des sciences du langage et des textes est connu, mais il n'est peut-être pas inutile de le rappeler. Un texte - un document, une archive, un récit du passé, un essai ou un traité écrit et publié dans un lointain passé - ne peuvent être appréhendés à bon escient si on ne tient compte de leur dimension langagière et argumentative. Pour dégager leurs contenus, il ne suffit pas de traverser le texte : on ne les appréhende pas en retrouvant un noyau dur sous sa gangue langagière. Le sens se construit toujours dans un échange verbal qui comprend des partenaires situés poursuivant leurs objectifs propres; il s'élabore dans l'épaisseur du discours, dans la mise en mots que règle la spécificité d'une interaction verbale. Il n'est donc pas possible de le dégager sans tenir compte de ces paramètres - car le même argument peut revêtir une signification très différente selon la façon dont il est formulé et développé, et selon l'auditoire auquel il est destiné dans des circonstances particulières. C'est donc pour étudier les textes et documents dont se nourrissent les sciences historiques (ou politiques, ou sociales, etc.) qu'il faut disposer des cadres et des instruments qui permettent de les analyser avec le plus de justesse et de finesse possible. Qui plus est, le discours est parfois lui-même Histoire, ou événement historique. C'est le cas, par exemple, du discours du Général de Gaulle du 18 juin 1940, dans son rapport à l'appel prononcé la veille par le Maréchal Pétain (Adam 1999 : 139-155).

Je vais essayer d'expliciter les différentes possibilités mentionnées ci-dessus à l'aide de quelques exemples, en insistant plus particulièrement sur l'apport spécifique de l'argumentation dans le discours au sein de l'espace global de l'AD. Faute de place, je me permettrai de renvoyer à des travaux que j'ai publiés ailleurs au cours des ans, mais aussi de m'appuyer sur des études menées par d'autres chercheurs et qui me semblent significatives, voire décisives, dans le domaine. Il s'agit bien sûr de simples indications données à titre d'exemplification, et non d'un panorama exhaustif.

\subsection{L'argumentation dans le discours dans l'espace des sciences du langage}

Dans l'espace des sciences du langage, le questionnement porte sur les moyens verbaux qui, au sein d'un fonctionnement discursif global, assurent à la parole son efficacité. Il s'agit donc d'explorer ces fonctionnements discursifs pour voir comment le discours permet au locuteur d'agir sur l'autre. 
31 Dans ce cadre, les acquis de la linguistique dans ses différents courants sont repris et réexaminés dans une perspective argumentative. Prenons, par exemple, les premiers travaux de Ducrot sur la présupposition. On se souvient que Ducrot, après avoir relevé les fonctions de l'implicite qui permettent de dire sans dire et de soustraire ainsi ce qu'on avance à la contradiction (1972:6), définit la présupposition et montre que "présupposer un certain contenu, c'est placer l'acceptation de ce contenu comme la condition du dialogue ultérieur » en transformant «du même coup les possibilités de parole de l'interlocuteur " (1972: 91). Le refus des présupposés amène une rupture de la communication. Il en ressort que la présupposition constitue pour le locuteur un moyen particulièrement efficace de faire accepter par son auditoire certaines vues qu'il ne soumet pas à son assentiment (il ne les "pose " pas) mais qu'il introduit comme allant de soi (il les "présuppose»). L'analyse argumentative peut exploiter les potentialités de l'étude menée en pragmatique intégrée en étudiant, par exemple, les "dessous » du débat politique télévisé (Amossy 1994). Elle peut aussi tirer profit des travaux entrepris sur l'implicite dans le domaine de la pragmatique, et en particulier dans l'ouvrage désormais classique de Kerbrat-Orecchioni (1986). Il est instructif, dans cette perspective, de dégager les fonctions de l'implicite lorsqu'il est sciemment mobilisé dans le discours de l'extrême-droite sur Israël et sur les juifs (Amossy 1999). Notons qu'on trouve des travaux nombreux et féconds sur les fonctions de l'implicite dans le discours argumentatif fondés tantôt sur les travaux de Grice, tantôt sur la notion rhétorique d'enthymème (comme syllogisme manquant).

Il est parfois nécessaire de faire le point sur les fonctions argumentatives de différents phénomènes qui n'ont pas fait l'objet d'une investigation dans le domaine des sciences du langage, et qu'il faut donc en un premier temps définir et décrire. Il en va ainsi du stéréotype dans sa définition de représentation collective figée (Amossy 1997) ou de la stéréotypie sous ses diverses formes (Amossy 2002, Amossy et Sternberg 2002) répertoriées par des disciplines comme la rhétorique (le topos rhétorique), la littérature (l'idée reçue), la stylistique (le cliché) (Amossy et Herschberg-Pierrot 1997). L'intégration de ces éléments dans une perspective discursive s'accompagne de l'exploration des différents rôles qu'ils peuvent jouer dans l'argumentation.

33 La recherche peut aussi se focaliser sur le profit que peut tirer l'argumentation de certains dispositifs d'énonciation. L'effacement énonciatif, qui fait actuellement l'objet de travaux importants dans les sciences du langage, autorise ainsi une mise en évidence des avantages que procure au locuteur la tentative de neutraliser sa parole en tentant de gommer aussi efficacement que possible sa subjectivité. S'appuyant sur les travaux de Vion, Alain Rabatel relève les marques formelles de l'effacement énonciatif pour l'articuler sur les effets d'argumentation indirecte qu'il permet (Rabatel 2004). Dans un autre domaine, l'étude de l'ethos rhétorique, reprise en $\mathrm{AD}$ à partir des travaux de Dominique Maingueneau, autorise également l'exploration de l'instance de locution dans l'échange verbal. Elle permet de montrer comment le locuteur construit une image de soi appropriée et efficace dans le rapport constitutif qu'il noue à l'allocutaire (Maingueneau dans Amossy 1999).

34 En l'occurrence, la reprise d'une notion rhétorique par les sciences du langage mène à une intégration de l'art de persuader antique dans une analyse argumentative qui se réclame de l'AD. Si l'ethos occupe actuellement une place prépondérante dans les sciences du langage, il faut bien voir que d'autres pôles sont également étudiés - en particulier le pathos ou la construction de l'émotion dans le discours qui permet 
d'emporter l'adhésion en touchant aussi bien le cœur que la raison de l'auditoire. On en donnera comme exemple les travaux de Christian Plantin, l'article de Patrick Charaudeau et le volume Les émotions dans les interactions dans lequel il a paru, ou encore le colloque tenu à Brest sur Le pathos en action (Rinn 2008). C'est dans cette optique également que l'on peut s'attacher, en les insérant dans une réflexion linguistique axée sur la force de la parole, à diverses notions rhétoriques - qu'elles soient puisées dans les figures et les tropes de l'elocutio (la prétérition, la digression, la métaphore, etc.) ou dans des catégories plus tardives comme les arguments en «ad» (ad populum, ad baculum, ad hominem). Christian Plantin a montré que l'étude des fallacies (paralogismes) participe d'un pôle formel de type évaluatif (la critique des arguments non-valides) auquel s'oppose un pôle langagier qui étudie l'argumentation dans des cadres interactifs (Plantin 1995 : 257). Plutôt que de rejeter la question des paralogismes, on peut alors l'inscrire dans la mouvance des études de l'argumentation en langue naturelle (Amossy 2006).

Il faut enfin mentionner un domaine important, qui relève également des sciences du langage et plus spécifiquement de l'AD: la description d'un genre de discours. L'argumentation apparaît alors comme une composante inhérente au fonctionnement de celui-ci. Il est possible de brasser large en explorant le fonctionnement du discours publicitaire (Adam et Bonhomme 1997), ou du discours politique dans ses différentes variétés (Charaudeau 2005, Schepens 2006). On peut aussi éclairer le fonctionnement d'un genre institué comme le pamphlet (Angenot 1982), la correspondance diplomatique (Cohen-Wiesenfeld 2004), ou l'entretien d'auteur (Yanoshevsky 2006). Ces études se greffent souvent sur des problématiques plus vastes - ainsi, l'analyse du pamphlet permet d'éclairer le fonctionnement de la parole polémique, celle de la correspondance diplomatique reprend la question de l'inscription de la subjectivité dans le discours en montrant comment se construit la dimension argumentative d'échanges épistolaires censés rester neutres, l'étude de l'entretien montre la coconstruction d'une image d'auteur dans la dynamique du dialogue. D'autres travaillent sur des genres non institués en examinant l'argumentation dans les échanges quotidiens: ainsi Guylaine Martel examine quelles stratégies argumentatives sont utilisées dans le discours oral spontané (1998).

La prise en compte du cadre générique montre à quel point l'art de persuader est déterminé par les règles et contraintes du genre de discours dans lequel il se déploie. C'est ainsi, par exemple, que l'argumentation politique d'olympe de Gouges qui, en tant que femme, n'avait guère accès à la prise de parole publique au début de la Révolution française, varie dans ses modalités en fonction des genres de discours qu'elle parvient à s'approprier (Siess 2005). De même, ce que Vera Brittain écrit concernant son vécu de la Grande guerre et le deuil de son fiancé, diffère lorsqu'elle écrit son journal intime et lorsqu'elle rédige des lettres à l'intention de son frère et de ses amis - pour ne pas mentionner le célèbre roman pacifiste publié dans les années 1930 (Amossy 2003). On peut voir ainsi comment la relation d'une même situation, ou la transmission d'une même thèse, revêt des formes différentes, voire acquiert un sens et un impact différent, lorsqu'elle se coule dans un format de communication et un dispositif d'énonciation particulier. 


\subsection{L'argumentation dans le discours et l'analyse textuelle}

Cependant, nous l'avons dit, le chercheur peut faire servir l'argumentation dans le discours à des buts qui relèvent d'autres disciplines que les sciences du langage, et répondent à d'autres besoins. En particulier, il lui est loisible de mobiliser ses cadres d'analyse pour étudier un texte ou un document particulier.

Ce besoin peut se faire sentir dans les sciences de la communication lorsqu'il s'agit de passer au crible un discours politique déterminé. Ainsi, on peut se pencher sur un discours de campagne de Nicolas Sarkozy ou de Ségolène Royal au moment des Présidentielles de 2007 en France, pour voir comment ils construisent un ethos: comment une femme peut projeter une image de présidentiable, ou selon quelles modalités il est donné à Sarkozy de gommer les aspects négatifs de son image préalable. On peut également examiner la manière dont des candidats aux Présidentielles de 2002, en l'occurrence Chirac et Le Pen, se sont emparés dans une interview particulière du thème de l'insécurité et ont exploité une doxa pour consolider leur ethos (Amossy 2005).

L'analyse argumentative met en évidence à la fois les objectifs du discours dans une situation de communication singulière et les stratégies déployées pour les réaliser dans leurs dimensions formelles et idéologiques. Aussi convient-elle également à l'analyse de l'archive qui retient l'attention de l'historien. Une analyse détaillée d'un texte publié le 14 novembre 1918 par Madeleine Vernet, «A la "Mère inconnue" du "Soldat inconnu" ", dans une revue intitulée La Mère éducatrice, montre ainsi comment l'auteur construit son auditoire féminin et tente d'emporter son adhésion à une protestation tout à fait minoritaire contre la cérémonie du Soldat Inconnu en particulier, et contre les guerres en général (Amossy 2006).

Cette démarche correspond également aux besoins des études littéraires, qui s'attachent tantôt à un texte bref dans son unicité, tantôt à une œuvre plus ou moins consacrée. Dans ce cadre, c'est la spécificité du texte, du genre dont il relève, de l'esthétique dans laquelle il s'inscrit ou qu'il contribue à élaborer, qu'il faut prendre en compte dans l'analyse. Ainsi, une étude d'un récit d'Henri Barbusse Ce qui fut sera à travers une analyse de son dispositif énonciatif et de sa facture analogique permet d'éclairer l'écriture pacifiste de Barbusse et son évolution (Amossy 2000). L'argumentation dans le discours permet aussi d'éclairer un texte testamentaire de Drieu la Rochelle (Amossy 2000), le discours pacifiste de Jacques dans Les Thibault de Roger Martin du Gard (Amossy 2000), ou encore les modalités argumentatives de l'ouverture de La porteuse de pain dans le cadre de l'esthétique du roman populaire de l'époque (Amossy 2007).

\subsection{L'argumentation dans le discours au service d'autres disciplines}

Cependant, l'argumentation dans le discours peut aussi être exploitée pour répondre de façon globale à des questions qui ne sont pas d'ordre linguistique, et qui se posent dans diverses disciplines des sciences humaines. Il ne s'agit pas dès lors de s'interroger sur les fonctions argumentatives de l'implicite, mais de voir dans quel mesure le discours du FN est antisémite. L'analyse du discours épistolaire et de la rhétorique des combattants n'est pas un but en soi, mais le moyen de comprendre comment les Poilus ont vécu la guerre, ce qui leur a permis de tenir aussi longtemps et dans quelle mesure 
il est légitime de parler de "consentement à la guerre" (Housiel). Ce n'est pas la construction de l'ethos en soi qui retient ici l'attention du chercheur, mais la question de savoir comment les étudiants français ont pu, pendant la guerre d'Algérie, modifier leur image pour la faire servir à la fois à leur revendication politique, et à un changement de leur propre statut (Orkibi). En d'autres termes, les cadres et les instruments de l'analyse du discours permettent d'éclairer un point particulier dont les enjeux se situent dans des disciplines extra-linguistiques.

Le chercheur doit alors constituer un corpus, au sens d'un ensemble de textes construit en fonction d'un paramètre qui leur confère une unité et permet de les soumettre à une même investigation. C'est cette option particulière qui se trouve en partie illustrée dans ce numéro sur L'analyse du discours au prisme de l'argumentation. Questions disciplinaires et interdisciplinaires.

Il faut bien voir, cependant, qu'une analyse à proprement parler linguistique peut également déboucher sur des questions médiatiques, politiques ou autres qui se posent dans d'autres disciplines. Ainsi une étude de la dénomination prise dans sa dimension argumentative permet non seulement d'explorer le fonctionnement de la dénomination comme telle, mais aussi de s'interroger sur l'objectivité revendiquée par la presse, et de travailler sur le traitement du terrorisme dans les journaux français des années 1980 (Koren 1996 : 205-258). En ce sens, la ligne de partage tracée entre les études disciplinaires et interdisciplinaire reste floue. Très souvent, une étude se propose aussi bien de mettre en évidence un fonctionnement discursif, que de traiter dans le corpus sélectionné une question de société. Réciproquement, un travail qui se focalise sur une question de société ou d'Histoire, ou encore d'esthétique littéraire, peut également mettre en lumière un fonctionnement discursif. C'est parce que l'analyse argumentative, comme l'AD dont elle relève, entend traiter des fonctionnements discursifs sur des cas concrets et non sur des exemples fabriqués, mais aussi parce qu'elle entend les examiner dans une situation de discours sociohistorique, qu'elle se situe nécessairement au carrefour des disciplines. Dans cette perspective, il est naturel que les interférences et les chevauchements entre les disciplines se multiplient - les tenants de l'AD et de l'argumentation faisant sans cesse incursion dans les domaines dans lesquels sont prélevés leurs exemples, les spécialistes des autres disciplines tendant de plus en plus fréquemment à se situer dans le cadre de l'AD et de l'argumentation pour explorer leur corpus et répondre aux questions qu'il suscite dans leur domaine propre ${ }^{6}$. Plutôt que d'y voir une confusion fâcheuse ou un estompement inquiétant des frontières, on peut se réjouir d'une interdisciplinarité qui s'est déjà à maintes reprises avérée fructueuse et qu'il convient, selon nous, de développer.

\section{BIBLIOGRAPHIE}

Adam, Jean-Michel. 1997. L'argumentation publicitaire. Rhétorique de l'éloge et de la persuasion (Paris : Nathan) 
Adam, Jean-Michel. 1999. Linguistique textuelle. Des genres de discours aux textes (Paris : Nathan) Adam, Jean-Michel et Heidmann, Ute (éds). 2005. Sciences du texte et analyse de discours. Enjeux d'une interdisciplinarité (Genève : Slaktine)

Amossy, Ruth et Herschberg Pierrot, Anne. 2005 [1997]. Stéréotypes et clichés. Langue, discours, société (Paris : Colin)

Amossy, Ruth (éd.). 1999. Images de soi dans le discours. La construction de l'ethos (Genève : Delachaux et Niestlé)

Amossy, Ruth et Maingueneau, Dominique (éds). 2004. L'analyse du discours dans les études littéraires (Toulouse : PUM)

Amossy, Ruth. 2006 [2000]. L'argumentation dans le discours (Paris : Colin)

Angenot, Marc. 1982. La parole pamphlétaire. Typologie des discours modernes (Paris : Payot)

Anscombre, Jean-Claude et Ducrot, Oswald. 1988. L'argumentation dans la langue (Liège : Mardaga)

Aristote. 1991. Rhétorique, trad. Ruelle, introd. M. Meyer, commentaire de B. Timmermans (Paris : Le livre de poche)

Bakhtine, Mikhail (Volochinov). 1977. Le marxisme et la philosophie du langage (Paris : Minuit)

Benveniste, Emile. 1974. Problèmes de linguistique générale, t. 2 (Paris : Gallimard)

Bordas, Eric (éd.). 2003. Ironies balzaciennes (Saint-Cyr : Pirot)

Breton, Philippe. 1996. L'argumentation dans la communication (Paris : La Découverte)

Breton, Philippe et Gauthier, Gilles. 2000. Histoire des théories de l'argumentation(Paris : La Découverte)

Burger, Marcel et Martel, Guylaine. 2005. Argumentation et communication dans les médias (Québec : Nota Bene)

Charaudeau, Patrick. 2005. Les médias et l'information. L'impossible transparence du discours (Bruxelles : de Boek)

Charaudeau, Patrick. 2005. Le discours politique. Les masques du pouvoir (Paris : Vuibert)

Charaudeau, Patrick et Maingueneau, Dominique (éds). 2002. Dictionnaire d'analyse du discours (Paris : Seuil)

Cohen-Wiesenfeld, Sivan. 2004. «L'inscription de la subjectivité dans le discours diplomatique », Semen 17

Ducrot, Oswald. 1972, Dire et ne pas dire (Paris : Hermann)

Ducrot, Oswald. 2004. « Argumentation rhétorique et argumentation linguistique », Doury, Marianne et Moirand, Sophie. (éds). L'argumentation aujourd'hui. Positions théoriques en confrontation (Paris : Presses de la Sorbonne Nouvelle)

Grize, Jean-Blaize. 1990. Logique et langage (Paris : Ophrys)

Kerbrat-Orecchioni, Catherine. 2002 [1980]. L'énonciation de la subjectivité dans le langage (Paris : Colin)

Kerbrat-Orecchioni, Catherine. 1986. L'implicite (Paris : Colin)

Kerbrat-Orecchioni, Catherine. 1990. Les interactions verbales, t. I (Paris : Colin) 
Koren, Roselyne. 1996. Les enjeux éthiques de l'écriture de presse ou la mise en mots du terrorisme (Paris : L'Harmattan)

Maingueneau, Dominique. 1998. Analyser les textes de communication (Paris : Nathan)

Maingueneau, Dominique. 2005. «L'analyse du discours et ses frontières », Marges linguistiques 9

Maingueneau, Dominique, 2006. Contre Saint-Proust ou la fin de la Littérature (Paris : Belin)

Martel, Guylaine, 1998. Pour une rhétorique du quotidien (Québec: CIRAL)

Meyer, Michel, 2005. Qu'est-ce que l'argumentation? (Paris : Vrin)

Oléron, Pierre, 1987. L'argumentation (Paris : PUF)

Perelman, Chaim et Olbrechts Tyteca, Lucie. 1970 [1958]. Traité de l'argumentation. . La nouvelle rhétorique (Bruxelles : Éditions de l'Université de Bruxelles)

Perelman, Chaim, 1977. L'empire rhétorique. Rhétorique et argumentation (Paris : Vrin)

Plantin, Christian. 1995. «L'argument du paralogisme », Hermès 15, « Argumentation et rhétorique I »

Plantin, Christian. 1996. L'argumentation (Paris : Le Seuil, « Mémo »)

Plantin, C., Doury, M. et Traverso, V. 2000. Les émotions dans les interactions (Arci/Presses universitaires de Lyon)

Plantin, Christian, 2005. L'argumentation (Paris : PUF, « Que sais-je?»)

Rabatel, Alain. 2004. «Effacement énonciatif et effets argumentatifs indirects dans l'incipit du Mort qu'il faut de Semprun », Semen 17

Rinn, Michael (éd.). 2008. Émotions et discours. L'usage des passions dans la langue (Rennes : Presses de l'Université de Rennes)

Semen 17, 2004. Amossy, R. et Koren, R. (éds). Argumentation et prise de position. Pratiques discursives Semen 21, 2006. Schepens. Philippe (éd.). Catégories pour l'analyse du discours politique Siess, Jürgen (éd.). 1998. La lettre entre réel et fiction (Paris : Sedes)

Siess, Jürgen et Valency, Gisèle (éds). 2002. La double adresse (Paris : L'Harmattan)

Siess, Jürgen, 2005. « Un discours politique au féminin : le projet d'Olympe de Gouges », Mots 78, pp. 9-21

Toulmin, S. E. 1993 [1958]). Les usages de l'argumentation (Paris : PUF)

Van Eemeren, Frans H., Grootendorst, Rob and Snoek Hoekemans, Francesca. 1996. Fundamentals of Argumentation Theory (NJ / London : Erlbaum)

Van Eemeren, Frans H. and Grootendorst, Rob. 1984. Speech Acts in Argumentative Discussions (Doordrecht : Foris)

Yanoshevsky, Galia. 2006. Les discours du Nouveau Roman. Essais, entretiens, débats (Lille : Septentrion)

\section{Etudes de Ruth Amossy citées à titre d'illustration}

1994. «Les dessous de l'argumentation dans le débat politique télévisé », Littérature, 93, «Le partage de la parole»

1999. « Israël et les juifs dans l'argumentation de l'extrême droite. Les fonctions du doxique et de l'implicite », Mots, 58, « Argumentations d'extrême droite » 
2000a. « Du témoignage au récit symbolique. Le récit de guerre et son dispositif énonciatif ", Milkovitch-Rioux, Catherine (éd.). Écrire la guerre. (Clermont-Ferrand: Presses universitaires Blaise Pascal)

2000b. « Plaidoirie et parole testamentaire. L'Exorde de Drieu la Rochelle », Kuperty-Tsur, Nadine (éd.) Écriture de soi et argumentation. Rhétorique et modèles de l'autoreprésentation (Caen : Presses Universitaires de Caen)

2000c. " Discours pacifiste et argumentation d'auteur dans L'Été 1914 de Roger Martin du Gard », Santa, Angels et Parra, Montse (éds). Relire L'Été 1914 et Épilogue de Roger Martin du Gard (Lleida : Pagès/El Fil d'Adriana)

2001. « Des topoi aux stéréotypes: le doxique entre logos et pathos », Eggs, Ekkehard (éd.). Topoi, discours, arguments, Zeitschrift für franzöische Sprache und Literatur (= Beiheft 32)

2003. «A Rhetorical Approach to Rewriting : Genre and Vera Brittain's Experience of Mourning », Journal of Romance Studies vol. III. 3 (Ben Porat, Ziva ed.)

2005. « Le maniement de la doxa sur le thème de l'insécurité : Chirac et Le Pen aux Présidentielles 2002 », Médias et Cultures, 1

2007. « Les récits médiatiques de grande diffusion au prisme de l'argumentation dans le discours : le cas du roman feuilleton ", Idéologie et stratégies argumentatives dans les récits imprimés de grande consommation. XIXe-XXIe siècles (Belphégor) http://etc.dal.ca/belphegor

\section{NOTES}

1. Malgré cette réserve sur la définition de la discipline, il me semble que les objectifs globaux et les positions théoriques de l'argumentation dans le discours restent assez proches de celles de charaudeau.

2. J'étudie la question des modalités argumentatives dans deux textes récents (2008): «Modalités argumentatives et registres discursifs : Le cas du polémique », Gaudin-Bordes, Lucile et Salvan, Geneviève (dir.). Les registres. Enjeux pragmatiques et visées stylistiques (Louvain-laNeuve: Academia-Bruylant) et «As modalidades argumentativas do discurso", Lara, G., Machado, I., Emediato, W. (orgs). Análuses do discuso hoje (Rio de Janeiro : Nova Fronteira).

3. Des données (D) sont avancées pour soutenir une conclusion (C), passage qui est autorisé par des garanties $(\mathrm{G})$ reposant sur un fondement (F - backing, B), et auquel peuvent s'appliquer des restrictions (R). Harry est né aux Bermudes (D), donc il est sujet Britannique (C) étant donné que ceux qui naissent aux Bermudes sont sujets Britanniques $(G)$ - sauf si ses parents ne l'étaient pas (R).

4. On trouvera un panorama de ces approches dans Breton, Philippe et Gauthier, Gilles. 2000. Histoire des théories de l'argumentation (Paris : La Découverte).

5. Sur le problème des frontières de l'AD dans le champ des sciences du langage, mais aussi dans son rapport avec d'autres disciplines, on lira l'indispensable réflexion de Dominique Maingueneau dans «L'analyse du discours et ses frontières » (2005).

6. Sans compter qu' « on peut difficilement soutenir que toute recherche sur le discours relève nécessairement d'une discipline. Pour nombre de travaux à visée fortement descriptive et/ou qui abordent des objets peu ou pas traités, on est dans l'incapacité de dire quelle discipline les régit. Les différences entre disciplines n'apparaissent en effet que si la recherche s'inscrit véritablement dans une problématique, profilée par l'intérêt qui gouverne la discipline concernée. » (Maingueneau 2005) 


\section{RÉSUMÉS}

L'objectif principal de cet article est double: exposer les avantages d'une intégration de l'argumentation dans l'AD (dans son versant français contemporain); traiter des questions d'interdisciplinarité issues de cette approche dans leurs dimensions à la fois scientifiques et institutionnelles. La première partie offre une synthèse de la théorie de « l'argumentation dans le discours " où l'argumentation en langue naturelle apparaît comme une partie intégrante d'un fonctionnement discursif global. Celui-ci doit être exploré dans sa situation de discours, son genre and ses aspects dialogiques, intertextuels et rhétoriques (ethos et pathos). Cette théorie se fonde sur une définition de l'argumentation empruntée à la nouvelle rhétorique de Perelman mais élargie - au-delà des discours à visée persuasive - aux discours qui, sans se targuer de persuader, déploient néanmoins une «dimension argumentative " qui contribue à faire voir le réel d'une certaine façon. La seconde partie interroge le statut disciplinaire de l'analyse du discours et de l'argumentation dans le discours comme une branche de la linguistique, d'une part, et comme une discipline autorisant des investigations littéraires, historiques, etc., d'autre part. Tout en exemplifiant la nature du type de questions étudiées dans chaque discipline, elle met en lumière les problèmes que suscite la transgression des limites disciplinaires traditionnelles. Elle suggère de faire la différence entre l'étude de questions strictement discursives propres aux sciences du langage, et la tentative de répondre à l'aide d'une analyse discursive et argumentative aux questionnements qui se font jour dans d'autres disciplines. Il apparaît néanmoins que la recherche fondée sur l'analyse de corpus éclaire souvent quelque aspect d'une discipline étrangère même quand elle met l'accent sur son questionnement propre.

The objective of this article is twofold: to present the advantages of integrating the study of argumentation into Discourse analysis (in its French contemporary developments); and to deal with the questions of interdisciplinarity derived from this approach, in both their scientific and institutional dimensions. The first part offers a synthesis of the "argumentation in discourse" theory where argumentation in natural language appears as part of an overall discursive system to be explored in its specific situation of discourse, its genre and its dialogical, intertextual and rhetorical aspects (ethos and pathos). This theory is based on a definition of argumentation borrowed from Perelman's New rhetoric but extended, beyond discourses clearly endowed with a persuasion aim, to types of discourses that do not intend to persuade and only display an "argumentative dimension": they contribute toward showing the surrounding world in a certain light. The second part questions the disciplinary status of discourse analysis and argumentation in discourse - as a branch of linguistics, on the one hand, and as a discipline authorizing literary or historical investigations, on the other hand. While exemplifying the nature of the issues explored in each case, it dwells on the institutional problems aroused by the transgression of traditional disciplinary boundaries. It suggests a division between studies of strictly discursive issues on the one hand, and attempts at answering through discursive, argumentative analysis diverse questions arising in other disciplines, on the other hand. It appears, however, that research based on case studies often highlights some aspect of another discipline even when putting the emphasis on its own questioning. 
INDEX

Keywords : argumentation, argumentative dimension, discourse analysis, historical case study, interdisciplinarity, literary studies

Mots-clés : analyse du discours, argumentation, dimension argumentative, étude littéraire, histoire, interdisciplinarité

\section{AUTEUR}

RUTH AMOSSY

Université de Tel-Aviv, ADARR 